\title{
Platelet rich plasma versus extracorporeal shock wave therapy in patients with non- calcific supraspinatous tears
}

\author{
Samah M. Alian ${ }^{1 *}$ (D), Rabab S. Zaghlol ${ }^{1}$ and Sahar S. Khalil ${ }^{1,2}$
}

\begin{abstract}
Background: To compare the clinical efficacy of platelet rich plasma (PRP) subacromial injection and extracorporeal shock wave therapy (ESWT) on refractory non-calcific partial thickness supraspinatous tendon tear.

Results: Significant improvement in all outcome measures achieved in both groups at 4 weeks follow-up period (the end of treatment course) $(P \leq 0.05)$. At 12 weeks follow-up period, group I, showed significant improvement in all outcome measures compared to baseline and group II; however, in group II, there was no significant difference compared to baseline; moreover, deterioration in the previous improvement was noticed.

Conclusion: Both PRP (group I) and ESWT (group II) are effective therapeutic methods in the management of refractory non-calcific partial thickness supraspinatous tendon tear; however, PRP (group I) has better long-term effects on both pain and function.
\end{abstract}

Keywords: Supraspinatous tear, Platelet rich plasma, Extracorporeal shock wave therapy

\section{Background}

Shoulder pain is a common health problem affecting up to half of the population at least once per year [1]. Rotator cuff consists of four tendons: supraspinatus, infraspinatus, subscapularis, and teres minor. Rotator cuff tendons arrange in a flat horseshoe flattened layer and converge together to inserts onto the head of the humerus [2]. Partial thickness rotator cuff tear (PTRCT) is caused by either intrinsic or extrinsic mechanisms; however, intrinsic mechanisms are much more predominant [3]. The supraspinatus tendon tear is the most common tendon involved in both partial and complete rotator cuff tears $[4,5]$.

Clinical suspicion of PTRCT should prompt imaging studies to disclose the location and the extent of the tear. Musculoskeletal ultrasound (MSUS) has been recently used for screening of rotator cuff tears; however, its value can be limited due to the difficulties to differentiate PTRCTs from

\footnotetext{
* Correspondence: dr_r18@yahoo.com

${ }^{1}$ Departments of Rheumatology, Physical Medicine and Rehabilitation,

Faculty of Medicine, Zagazig University, Zagazig, Egypt

Full list of author information is available at the end of the article
}

scars in tendons or tiny focal full thickness rotator cuff tear $[2,6]$. Using expert hands, ultrasonography could reach similar efficacy of MRI in detecting PTRCT $[7,8]$.

The optimum management of PTRCT is multifactorial and guided by the patient's related factors such as age, general condition, and functional level before the injury. A high percentage of partial rotator cuff tears involve less than half thickness of the whole rotator cuff; consequently, it does not usually lead to retraction of the muscle. The treatment always starts conservatively with different options are available; rest, non-steroidal anti-inflammatory drugs, therapeutic local injections, physiotherapy, nerve blocks, and platelet rich plasma (PRP) injection [2, 3]. Non-operative conservative options are likely to be more effective in younger patients with PRCTs [9].

The pathogenesis of rotator cuff tear is still under debate. The rotator cuff has weak regeneration ability due to the poor tendon's vascular supply. PRP is the bioactive portion of the whole blood that has been recently used in several aspects of regenerative medicine, as it 
aids in the promotion of healing by stimulating angiogenesis and cell proliferation $[10,11]$.

Extracorporeal shock wave therapy (ESWT) is also a recent modality that is currently used in the rehabilitation of various musculoskeletal conditions [12]. ESWT might be considered as an alternative therapy for the patient with an ineffective conservative treatment or prior to operative management of rotator cuff disorders. The efficacy of using ESWT in rotator cuff disorders is still controversial [13].

ESWT can enhance tissue regeneration and promote neovascularization, so it could endorse tendon healing after injury. Moreover, ESWT could be an effective tool to induce relaxation of tightened disorganized tendon fibers; thus, reduces pain and increases the range of motion of the affected shoulder immediately post-treatment [14].

In this study, we compared the therapeutic effects of ESWT versus PRP therapy in treating refractory noncalcific partial supraspinatous tendon tear (as it is the most common one in rotator cuff tears) in order to establish a more effective management plan.

\section{Methods \\ Patients}

This study is a prospective randomized controlled study that included 30 patients, who complained of chronic shoulder pain (> 6 months) with ultrasonography evidence of non-calcific partial thickness supraspinatus tendon tear. The cases were either referred or primary diagnosed in rheumatology, physical medicine, and rehabilitation department, university hospitals from December 2017 to December 2018. Screening physical, laboratory, and detailed ultrasonographic shoulder evaluations were conducted to select the patients who met the inclusion criteria (Table 1). Patients were randomly selected and allocated into two parallel therapy groups using random allocation software.

Detailed physical examinations and demographic features were recorded for each patient. Routine laboratory investigations such as complete blood count (CBC) and erythrocyte sedimentation rate (ESR) were performed. Informed consent was taken from each participant before entering the study. The study was approved by the ethics committee of the university and was conducted in accordance with the declaration of Helsinki.

\section{Imaging}

All patients were examined by one experienced physiatrist with more than 5 years of experience in the MSUS field. B-Mode (grey-scale) real-time ultrasound was performed using (Hitachi-Aloka F37, Japan interfaced with a $10-18 \mathrm{MHz}$ linear array transducer). Rotator cuff abnormalities were defined as follows: "normal, tendinosis, partial-thickness tear, or full-thickness tear". If partial thickness supraspinatous tendon tear was detected on
Table 1 Eligibility criteria

Criteria

Inclusion criteria

Patients were included in the study if they had all of the following:

Shoulder pain for more than 6 months

Shoulder pain scores more than 5 on a numeric rate scale for pain of 0 10.

Diagnosed clinically as RC tear, painful mid-arc and/or positive HawkinsKennedy, drop arm, empty can, and lateral jobe tests [15-17].

Diagnosed with non-calcific supraspinatous partial thickness tear of less than $50 \%$ of the tendon thickness upon sonographic examination.

No or little response to conservative treatment for 3 months (one course of NSAIDs, one course of 3 weeks physiotherapy, and one local corticosteroid injection).

Exclusion criteria

Patients were excluded in the study if they had any of the following:

Obvious pathology of the shoulder, such as calcified tendon by US examination, full thickness tear, partial tear equal or greater than $50 \%$ of the tendon thickness, tear of other rotator cuff tendons rather than supraspinatous tendon.

Inflammatory, advanced degenerative, or septic arthritis of the shoulder Referred and radiculopathy pain.

Previous surgery to the same shoulder.

NSAID use in the past 2 weeks or corticosteroid injection within 6 weeks before the study.

The unstable medical condition, a known uncontrolled systemic disease, cardiac pacemaker, pregnancy, or psychiatric problems.

Thrombocytopenia $(<150,000$ platelets $/ \mathrm{ml})$, anti-platelet and anticoagulant medications, platelet/bleeding disorders.

Refused or unable to sign a consent

sonographic examination, then it is documented as follows: "its location (tear site in the articular surface, bursal surface or intra-substance) and percentage of tendon thickness involvement (less or more than 50\%)." Moreover, any other abnormalities in the shoulder joint or other tendons were documented. In this study, only patients with non-calcific tears of less than half thickness of the supraspinatus tendon were included (Fig. 1a-d).

\section{Grouping and interventions}

Group I consisted of 15 patients who had received two PRP injections, with 4-weeks interval, into subacromial space (targeted to the tear site), using ultrasound guidance under complete aseptic technique. PRP was prepared by the following method: $10 \mathrm{ml}$ of the patient's blood was collected and mixed with $1 \mathrm{mg} / \mathrm{mL}$ ethylenediaminetetraacetic acid anticoagulant (EDTA). Then, it was placed in centrifuge first spin at a force of $1600 \times g$ for $10 \mathrm{~min}$ to separate erythrocytes from the plasma. After that, the obtained plasma was centrifuged again for a second spin at a force of $2000 \times g$ for another $10 \mathrm{~min}$ to separate the platelet poor plasma (PPP) from the platelet rich plasma (PRP). 


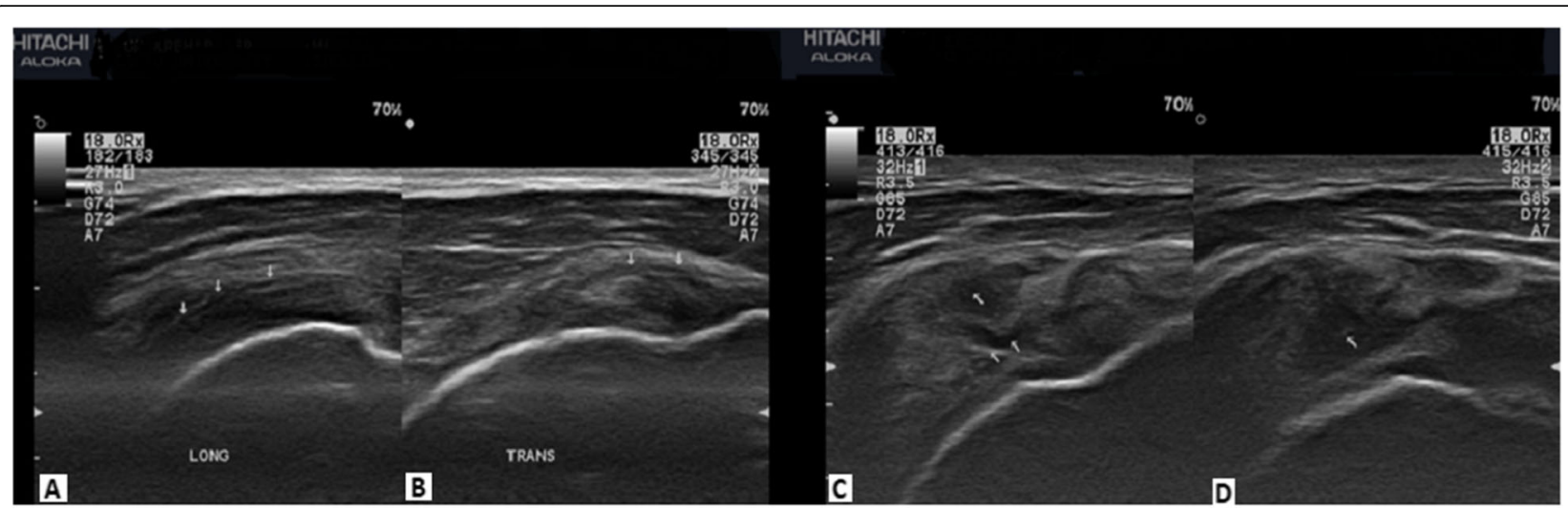

Fig. 1 Articular side partial tear in supraspinatus tendon seen at both longitudinal (a) and transverse views (b). An interstitial partial tear in supraspinatus tendon seen at both longitudinal (c) and transverse (d) views

Finally, the obtained PRP is about $2-3 \mathrm{ml}$ [18]. In case of any post-injection pain, only cold packs and acetaminophen were allowed to use in the first week of injection.

Group II consisted of 15 patients who were received four sessions of extracorporial shock wave therapy (ESWT) using (Intelect Mobile. 38. RPW, Chattanooga, Guildford Surrey, UK), with a frequency of once a week for four consecutive weeks. Three thousand radial shocks at an energy flux density of $0.068 \mathrm{mg} / \mathrm{mm}^{2}$ were applied each session to an area $1 \mathrm{~cm}$ proximal to the insertion of the tendon in the bone.

A self-exercise protocol (home based program) was demonstrated to all patients of both groups to improve range of motion (ROM) and strengthen the rotator cuff to improve muscle control and maintain the function of the affected shoulder [19]. They were allowed to resume usual daily living activities; however, overhead activity and heavy lifting vigorous lifting were prohibited.

\section{Evaluation of treatment efficacy}

An independent physician who was blinded to group allocation throughout the study had performed all evaluations. All patients were evaluated initially, then after 4 and 12 weeks of therapy with the following parameters:

\section{Numeric rating scale (NRS) of shoulder pain}

It is a self-reporting tool for evaluating the pain severity. Patients point to their amount of pain on a scale of zero (no pain) to 10 (agonizing severe pain), according to their subjective feelings on a $10-\mathrm{mm}$ scale drawn on paper. In this scale, the higher the score indicates the more pain experienced [20].

\section{Shoulder pain and disability index (SPADI)}

- It assesses the level of shoulder pain and disability. It consists of 5 items for pain measuring and 8 items for measuring of disability, each subscale is summated and converted to a score out of 100; a higher score indicates greater impairment/disability [21].

\section{- Active shoulder abduction degree measured by the goniometer.}

\section{Statistical analysis}

All data were collected and analyzed using the Statistical Package for Social Sciences (SPSS) version 24. Descriptive statistics including means and standard deviations (SD) were performed. Comparison of qualitative data between groups was performed using the "Chi-square test". Comparison of quantitative data between groups both parametric and non-parametric distribution using "independent t-test or Mann-Whitney test" respectively. The continuous variables across time were compared using "paired t-test or Wilcoxon Signed-Rank Test. All tests were two-sided differences were considered statistically significant when P-values were $\leq 0.05$.

\section{Results}

No significant differences were found in the baseline characteristics of the study population (both groups) including age, sex, pain duration, affected side between the PRP group and the ESWT group at baseline $(p>0.05)$ (Table 2).

By ultrasound examination, most of the tears were found at the articular surface $53.3 \%$ in group I and 66.7 $\%$ in group II and to a lesser extent the bursal and interstitial tears (20\% group I, 20\% group II, and $26.7 \%$ group I, $13.3 \%$ group II respectively), and the most common associated shoulder lesion by MSUS examination is subacromial bursitis (60\%) in group I and (46.7\%) in group II. There were no significant differences in the initial ultrasound grey-scale findings between both groups at baseline evaluation $(p>0.05)$ (Table 3).

After 4 weeks of therapy, we found that all the parameters showed a real improvement in all patients of both groups compared to baseline $(P \leq 0.05)$. When comparing 
Table 2 Baseline characteristics of both groups

\begin{tabular}{llll}
\hline Baseline characteristics & $\begin{array}{l}\text { Group 1 } \\
(n=15)\end{array}$ & $\begin{array}{l}\text { Group II } \\
(n=15)\end{array}$ & $P$ \\
\hline Age (years), mean \pm SD & $40.13 \pm 8.46$ & $39.20 \pm 9.16$ & 0.095 \\
Male: Female, $n(\%)$ & $9(60): 6(40)$ & $9(60): 6(40)$ & 1.000 \\
Pain duration (months), mean \pm SD & $7.86 \pm 1.92$ & $8.13 \pm 2.16$ & 0.68 \\
Side affected, dominant: non-dominant, $n(\%)$ & $11(73.3): 4(26.7)$ & $10(66.7): 5(33.3)$ & 0.69 \\
\hline
\end{tabular}

the improvements in both groups, we found no significant difference between them in all the evaluated variables (VAS, SPADI and active abduction degree) (Table 4).

After 12 weeks of therapy, group I patients showed improvement in all the evaluated variables was significant compared to baseline and group II, but we found no significant difference in the outcome measures compared to 4 weeks follow-up. In group II, there was no significant difference compared to baseline however, significant deterioration was found in the previous improvements found in the four weeks follow-up in all the outcome measures. Therefore, the PRP treated group showed significantly better outcomes at the 12 weeks follow-up than the ones observed in the ESWT treated group. No significant complications were encountered secondary to ESWT or PRP injection therapy. Patients had tolerated both well and no withdrawals of the study were encountered (Table 4).

\section{Discussion}

This study was conducted to compare the therapeutic effectiveness of PRP injections versus ESWT on refractory shoulder pain associated with non-calcific partial thickness supraspinatous tendon tear. In our study, the patients were followed-up for 12 weeks in order to detect any recurrence of symptoms and to ascertain the long-term effectiveness of the therapies.

In this study, we found the most frequent tears were at the articular footprint of the supraspinatus tendon. This finding was confirmed in many other studies [22-24].
Moreover, our data showed that both PRP injections and ESWT were effective in the treatment of pain and dysfunction. However, the PRP treated group I had continuous improvements at the 12 weeks follow-up tested period, while the ESWT group showed relapse.

Our result matched with that of Unlu et al. [25], which showed that injection of PRP around the tendon had beneficial clinical effects on improving subjective pain in patients who have tendinosis and tendon tear, with excellent tolerance.

Another study investigated the effect of ultrasonographic guided PRP shoulder injection in patients with < $1 \mathrm{~cm}$ PTRCT and poor response to conservative treatments, they concluded that only one PRP injection is effective to decrease pain, improve shoulder function and range of motion [26]. Moreover, these results were consistent with that of Scarpone et al. [27], who reported that a single local injection of PRP under ultrasound guidance had resulted in sustained improvement in clinical, functional and radiologic (MRI) outcomes in patients with refractory rotator cuff tears. They attributed the improvement to the potential healing enhancement capability of PRP on torn rotator cuff tendons.

A recent large-scale prospective, randomized, controlled study was conducted on 184 patients with rotator cuff tears. They concluded that PRP is effective in inducing healing of small to medium rotator cuff tears [28]. Moreover, the effect of PRP on PRCT was confirmed in another case series study [29].

In the study of Rha et al. [30], who compared dry needling to two PRP injections with four weeks interval in patients with rotator cuff disease and found that PRP

Table 3 Initial grey-scale ultrasound findings in both groups

\begin{tabular}{|c|c|c|c|c|c|}
\hline Variable & & Group $1(n=15)$ & Group II $(n=15)$ & $x^{2}$ & P \\
\hline \multirow[t]{3}{*}{ Tear site, $n(\%)$} & Bursal surface & $3(20.0)$ & $3(20.0)$ & 0.89 & 0.64 \\
\hline & Articular surface & $8(53.3)$ & $10(66.7)$ & & \\
\hline & Interstitial & $4(26.7)$ & $2(13.3)$ & & \\
\hline \multirow[t]{5}{*}{ Associated lesions, $n$ (\%) } & Subacromial bursitis & $9(60.0)$ & $7(46.7)$ & 0.54 & 0.46 \\
\hline & SS tendinopathy & $8(53.3)$ & $10(66.7)$ & 0.56 & 0.46 \\
\hline & Bicipital tendinitis & $7(46.7)$ & $5(33.3)$ & 0.56 & 0.46 \\
\hline & ACJ arthrosis & $6(40.0)$ & $5(33.3)$ & 0.14 & 0.70 \\
\hline & GH joint effusion & $5(33.3)$ & $7(46.7)$ & 0.56 & 0.46 \\
\hline
\end{tabular}

SS supraspinatous, $A C J$ acromioclavicular joint, $G H$ glenohumeral 
Table 4 Outcome measures before and after treatment ( 4 and 12 weeks) in both groups

\begin{tabular}{|c|c|c|c|c|c|c|c|}
\hline Scales mean \pm SD & Groups & $\begin{array}{l}\text { Baseline } \\
(0 \text { weeks })\end{array}$ & 4 weeks & 12 weeks & $\begin{array}{l}P \text { value }{ }^{2} \\
\text { (4 vs. } 0 \text { weeks) }\end{array}$ & $\begin{array}{l}P \text { value }{ }^{2} \\
(12 \text { vs. } 0 \text { weeks })\end{array}$ & $\begin{array}{l}P \text { value }^{2} \\
\text { (12 vs. } 4 \text { weeks) }\end{array}$ \\
\hline NRS for pain & $\begin{array}{l}\text { II } \\
P \text { value }\end{array}$ & $\begin{array}{l}6.86 \pm 1.64 \\
7.20 \pm 1.89 \\
0.56\end{array}$ & $\begin{array}{l}3.06 \pm 1.48 \\
3.86 \pm 0.99 \\
0.180\end{array}$ & $\begin{array}{l}3.26 \pm 1.98 \\
6.66 \pm 2.46 \\
<0.01^{*}\end{array}$ & $\begin{array}{l}<0.001^{*} \\
<0.000^{*}\end{array}$ & $\begin{array}{l}<0.001^{*} \\
0.18\end{array}$ & $\begin{array}{l}0.18 \\
<0.001^{*}\end{array}$ \\
\hline SPADI & $\begin{array}{l}\text { II } \\
P \text { value }\end{array}$ & $\begin{array}{l}89.80 \pm 20.88 \\
86.40 \pm 20.21 \\
0.61\end{array}$ & $\begin{array}{l}38.13 \pm 20.53 \\
45.46 \pm 11.70 \\
0.11\end{array}$ & $\begin{array}{l}39.73 \pm 24.17 \\
80.40 \pm 26.15 \\
<0.01^{*}\end{array}$ & $\begin{array}{l}<0.001^{*} \\
<0.000^{*}\end{array}$ & $\begin{array}{l}<0.001^{*} \\
0.18\end{array}$ & $\begin{array}{l}0.18 \\
<0.000^{*}\end{array}$ \\
\hline Abduction & $\begin{array}{l}\text { II } \\
P \text { value }\end{array}$ & $\begin{array}{l}89.93 \pm 6.21 \\
90.80 \pm 8.07 \\
0.71\end{array}$ & $\begin{array}{l}152.13 \pm 21.08 \\
149.40 \pm 9.81 \\
0.66\end{array}$ & $\begin{array}{l}151.13 \pm 23.67 \\
99.93 \pm 26.44 \\
<0.001^{*}\end{array}$ & $\begin{array}{l}<0.000^{*} \\
<0.000^{*}\end{array}$ & $\begin{array}{l}<0.000^{*} \\
0.17\end{array}$ & $\begin{array}{l}0.19 \\
<0.000^{*}\end{array}$ \\
\hline
\end{tabular}

NRS numeric rate scale, SPADI shoulder pain and disability index

${ }^{*} P<0.05$ : significant

$P$ value ${ }^{1}$ : significance between groups

$P$ value $^{2}$ : significance in the same group at the follow-up

injections were more effective and on symptoms and function than dry needling at six months post-therapy.

Regarding ESWT, the present results were consistent with another study that analyzed the effect of ESWT in patients with non-calcific supraspinatus tendinopathy. They reported significant improvement of shoulder pain and functional gain after treatment with ESWT [31].

In the study done by Chen et al. [14], who studied the effect of combined ESWT, eccentric exercise, and conventional therapy compared to conventional therapy alone in patients with non-calcific rotator cuff tendinopathy. Their results implied a superior result of combination therapy and suggested that adding shock wave therapy had significant effects on the management of non-calcific rotator cuff tendinopathy.

Moreover, our results were consistent with a study done by Chou et al. [32], who assessed the clinical outcomes of using ESWT for patients with refractory tendinitis or partial tear of the rotator cuff tendon in the athletic and the non-athletic groups, they found that ESWT was equally effective in both groups with a high level of satisfaction.

Also, as regarding ESWT Zamzam et al. [33], who studied the effect of ESWT versus ultrasound-guided steroid injection in the treatment of 30 patients with calcific and noncalcific supraspinatus tendinopathy for more than 3 months. They found statistically significant improvement in pain relief and clinical examination parameters, but there was no statistically significant difference between the two groups in the treatment of chronic supraspinatus tendinopathy.

To the best of our knowledge, this is the first study comparing the therapeutic effects of PRP versus ESWT for refractory non-calcific partial thickness supraspinatous tendon tear. However, regarding this combination, a randomized trial done by Vetrano et al. [34], included a total of 46 patellar tendinopathy patients. They compared the effects of two every other week injections of PRP and three ESWT therapy sessions. They found that both groups showed significant improvement of symptoms at all follow-up periods, but the PRP group had significantly better long-term improvement.

Furthermore, another randomized controlled trial conducted by Smith and Sellon [35], included a comparative analysis of the outcome of treatment by PRP and ESWT in athletes with chronic patellar tendinopathy and they found a positive response in both PRP injection and ESWT groups. However, the PRP-treated patients reported significantly greater improvements in pain relief at 6 months to follow-up and significantly better functional outcomes and satisfaction at 12 months follow-up.

Until now, there is no standardized guideline that clearly defines the number, protocol, interval and frequency of treatments needed for execution of either PRP therapy or ESWT, as regards side effects in general ESWT is more safe than PRP as considering the general side effects of local injection like infection, but in our study we did not record any side effects from both lines of treatment, also in our study PRP has nearly half the numbers of patient visits (2) in comparison to ESWT (4) and both nearly have the same cost.

The limitations of this study include the short-term period of follow-up and the small number of participants. Therefore, continuous efforts to establish the most effective management tools are needed in the future. Further studies with a larger number of participants and longer follow-up periods will be needed.

\section{Conclusion}

Both PRP (group I) and ESWT (group II) are effective therapeutic methods in the management of refractory non-calcific partial thickness supraspinatous tendon tear; however, PRP (group I) has better long-term effects on both pain and function.

\section{Abbreviations}

PRP: Platelet rich plasma; ESWT: Extracorporeal shock wave therapy; PTRCT: Partial thickness rotator cuff tear; MSUS: Musculoskeletal ultrasound; NRS: Numeric rating scale; SPADI: Shoulder pain and disability index 


\section{Acknowledgements}

No specific acknowledgements.

\section{Authors' contributions}

SA had contributed to the conception, design of the work; did the ultrasonography examination, and platelet rich plasma injection guided by ultrasound, the acquisition, analysis, interpretation of data; had drafted the work and substantively revised it, also, had approved the submitted version (and any substantially modified version) and finally, had agreed both to be personally accountable for her own contributions and to ensure that questions related to the accuracy or integrity of any part of the work, even ones in which she was not personally involved, are appropriately investigated, resolved, and the resolution documented in the literature. RZ had contributed to the conception, design of the work; the acquisition, analysis, interpretation of data; had drafted the work and substantively revised it, also, had approved the submitted version (and any substantially modified version) and finally, had agreed both to be personally accountable for her own contributions and to ensure that questions related to the accuracy or integrity of any part of the work, even ones in which she was not personally involved, are appropriately investigated, resolved, and the resolution documented in the literature. SK author had contributed to the conception, design of the work; the acquisition, analysis, interpretation of data; had drafted the work and substantively revised it, also, had approved the submitted version (and any substantially modified version) and finally, had agreed both to be personally accountable for her own contributions and to ensure that questions related to the accuracy or integrity of any part of the work, even ones in which she was not personally involved, are appropriately investigated, resolved, and the resolution documented in the literature. All authors have read and approved the manuscript.

\section{Funding}

No fund for this article.

\section{Availability of data and materials}

Not applicable

\section{Ethics approval and consent to participate}

Written informed consent was obtained from patients for their study participation.

\section{Consent for publication}

Written informed consent was obtained from patients for publication of this work.

\section{Competing interests}

The authors declare that they have no competing interests.

\section{Author details}

'Departments of Rheumatology, Physical Medicine and Rehabilitation, Faculty of Medicine, Zagazig University, Zagazig, Egypt. ${ }^{2}$ Department of Physical Medicine and Rehabilitation, Dubai Hospital, Dubai, United Arab Emirates.

Received: 13 May 2020 Accepted: 31 May 2020

Published online: 22 September 2020

\section{References}

1. Luime JJ, Koes BW, Hendriksen IJ (2004) e. Prevalence and incidence of shoulder pain in the general population; a systematic review. Scand $J$ Rheumatol 33(2):73-81

2. Matthewson G, Beach CJ, Nelson AA, Woodmass JM, Ono Y, Boorman RS, Lo IK, Thornton GM (2015) Partial thickness rotator cuff tears: current concepts. Advances in orthopedics 2015

3. Shin KM (2011) Partial-thickness rotator cuff tears. Korean J Pain 24(2):69-73

4. Tuite MJ (2012) Magnetic resonance imaging of rotator cuff disease and external impingement. Magn Reson Imaging Clin N Am 20(2):187-200

5. Freygant M, Dziurzyńska-Białek E, Guz W et al (2014) Magnetic resonance imaging of rotator cuff tears in shoulder impingement syndrome. Pol J Radiol 79:391-397
6. Hinsley H, Nicholls A, Daines M, Wallace G, Arden N, Carr A (2014) Classification of rotator cuff tendinopathy using high definition ultrasound. Muscles Ligaments Tendons J 4(3):391

7. Teefey SA, Rubin DA, Middleton WD, Hildebolt CF, Leibold RA, Yamaguchi K (2004) Detection and quantification of rotator cuff tears: comparison of ultrasonographic, magnetic resonance imaging, and arthroscopic findings in seventy-one consecutive cases. JBJS. 86(4):708-716

8. De Jesus JO, Parker L, Frangos AJ, Nazarian LN (2009) Accuracy of MRI, MR arthrography, and ultrasound in the diagnosis of rotator cuff tears: a metaanalysis. Am J Roentgenol 192(6):1701-1707

9. Maman E, Harris C, White L, Tomlinson G, Shashank M, Boynton E (2009) Outcome of nonoperative treatment of symptomatic rotator cuff tears monitored by magnetic resonance imaging. JBJS. 91(8):1898-1906

10. Castricini R, Longo UG, De Benedetto M, Panfoli N, Pirani P, Zini R, Maffulli N, Denaro V (2011) Platelet-rich plasma augmentation for arthroscopic rotator cuff repair: a randomized controlled trial. Am J Sports Med 39(2): 258-265

11. Sánchez M, Anitua E, Orive G, Mujika I, Andia I (2009) Platelet-rich therapies in the treatment of orthopaedic sport injuries. Sports Med 39(5):345-354

12. Romeo P, Lavanga V, Pagani D, Sansone V (2014) Extracorporeal shock wave therapy in musculoskeletal disorders: a review. Med Princ Pract 23(1):7-13

13. Moya D, Ramón S, Guiloff L, Gerdesmeyer L (2015) Current knowledge on evidence-based shockwave treatments for shoulder pathology. Int J Surg 24:171-178

14. Chen TW, Huei Su J, Lin TY, Lin CW, Chou PS (2017) Effects of eccentric exercise and extracorporeal shock wave therapy on rehabilitation of patients with noncalcific rotator cuff tendinopathy. Clin Res Foot Ankle 5(222):2

15. Hawkins RJ, Kennedy JC (1980) Impingement syndrome in athletes. Am J Sports Med 8(3):151-158

16. Jain NB, Wilcox RB III, Katz JN, Higgins LD (2013) Clinical examination of the rotator cuff. PM\&R. 5(1):45-56

17. Gillooly JJ, Chidambaram R, Mok D (2010) The lateral Jobe test: a more reliable method of diagnosing rotator cuff tears. Int J Shoulder Surg 4(2):41

18. Araki J, Jona M, Eto H, Aoi N, Kato H, Suga H, Doi K, Yatomi Y, Yoshimura K (2011) Optimized preparation method of platelet-concentrated plasma and noncoagulating platelet-derived factor concentrates: maximization of platelet concentration and removal of fibrinogen. Tissue Eng Part C Methods 18(3):176-185

19. Heers G, Anders S, Werther M, Lerch K, Hedtmann A, Grifka J (2005) Efficacy of home exercises for symptomatic rotator cuff tears in correlation to the size of the defect. Sportverletzung Sportschaden: Organ der Gesellschaft fur Orthopadisch-Traumatologische Sportmedizin 19(1):22-27

20. Hawker GA, Mian S, Kendzerska T, French M (2011) Measures of adult pain: visual analog scale for pain (vas pain), numeric rating scale for pain (nrs pain), mcgill pain questionnaire (mpq), short-form mcgill pain questionnaire (sf-mpq), chronic pain grade scale (cpgs), short form-36 bodily pain scale (sf-36 bps), and measure of intermittent and constant osteoarthritis pain (icoap). Arthritis Care Res 63(S11):S240-S252

21. Heald SL, Riddle DL, Lamb RL (1997) The shoulder pain and disability index: the construct validity and responsiveness of a region-specific disability measure. Phys Ther 77(10):1079-1089

22. Modi CS, Smith CD, Drew SJ (2012) Partial-thickness articular surface rotator cuff tears in patients over the age of 35: etiology and intra-articular associations. Int J Shoulder Surg 6(1):15-18

23. Fukuda $\mathrm{H}$ (2003) The management of partial-thickness tears of the rotator cuff. J Bone Joint Surg (Br) 85:3-11

24. Lehman RC, Perry CR (2003) Arthroscopic surgery for partial rotator cuff tears. Arthroscopy. 19:E81-E84

25. Unlu MC, Kivrak A, Kayaalp ME, Birsel O, Akgun I (2017) Peritendinous injection of platelet-rich plasma to treat tendinopathy: a retrospective review. Acta Orthop Traumatol Turc 51 (6):482-487

26. Tahririan MA, Moezi M, Motififard M, Nemati M, Nemati A (2016) Ultrasound guided platelet-rich plasma injection for the treatment of rotator cuff tendinopathy. Adv Biomed Res 5

27. Scarpone M, Rabago D, Snell E, Demeo P, Ruppert K, Pritchard P, Arbogast G, Wilson JJ, Balzano JF (2013) Effectiveness of platelet-rich plasma injection for rotator cuff tendinopathy: a prospective open-label study. Global Adv Health Med 2(2):26-31

28. Cai Y, Sun Z, Liao B, Zhanqiang S, Xiao T, Zhu P (2019) Sodium hyaluronate and platelet-rich plasma for partial-thickness rotator cuff tears. Med Sci Sports Exerc 51(2):227-233 
29. Zafarani Z, Mirzaee F, Guity M, Aslani H (2017) Clinical results of platelet-rich plasma for partial thickness rotator cuff tears: a case series. Arch Bone Jt Surg 5(5):328-331

30. Rha DW, Park GY, Kim YK, Kim MT, Lee SC (2013) Comparison of the therapeutic effects of ultrasound-guided platelet-rich plasma injection and dry needling in rotator cuff disease: a randomized controlled trial. Clin Rehabil 27(2):113-122

31. Galasso O, Amelio E, Riccelli DA, Gasparini G (2012) Short-term outcomes of extracorporeal shock wave therapy for the treatment of chronic non-calcific tendinopathy of the supraspinatus: a double-blind, randomized, placebocontrolled trial. BMC Musculoskelet Disord 13(1):86

32. Chou WY, Wang CJ, Wu KT, Yang YJ, Cheng JH, Wang SW (2018) Comparative outcomes of extracorporeal shockwave therapy for shoulder tendinitis or partial tears of the rotator cuff in athletes and non-athletes: retrospective study. Int J Surg 51:184-190

33. Zamzam M, El Yasaki A, ElGarabawy N, El Ghandour LE (2019) Shockwave therapy versus local steroid injection in chronic supraspinatus tendinopathy. Egypt Rheumatol Rehabil 46:141-147

34. Vetrano M, Castorina A, Vulpiani MC, Baldini R, Pavan A, Ferretti A (2013) Platelet-rich plasma versus focused shock waves in the treatment of jumper's knee in athletes. Am J Sports Med 41(4):795-803

35. Smith J, Sellon JL (2014) Comparing PRP injections with ESWT for athletes with chronic patellar tendinopathy. Clin J Sport Med 24(1):88-89

\section{Publisher's Note}

Springer Nature remains neutral with regard to jurisdictional claims in published maps and institutional affiliations.

\section{Submit your manuscript to a SpringerOpen ${ }^{\circ}$ journal and benefit from:}

- Convenient online submission

- Rigorous peer review

- Open access: articles freely available online

- High visibility within the field

- Retaining the copyright to your article

Submit your next manuscript at $\boldsymbol{\nabla}$ springeropen.com 